\title{
Brain Activities supporting Finger Operations, analyzed by Neuro-NIRS,
}

\author{
Miki FUCHIGAMI $^{1}$, Akira OKADA ${ }^{1}$, Hiroshi TAMURA ${ }^{2}$ \\ ${ }^{1}$ Osaka City University, Sugimotocho, Osaka City, Japan \\ 2 Institute for HUMAN INTERFACE, C1803 Portpia plaza, 6-14 Minatojima nakamachi, \\ Chuoku, Kobe City, JAPAN 650-0046 (tamura@mobilergo.com)
}

\begin{abstract}
Brain activities are analyzed for the button selection task using Neuro-NIRS, which is analysis method of quick component of NIRS. Quick component of NIRS is numerically quantifies by short time average of absolute value. The average is represented by, maximum and density. Since button selection task is short time event, it seems the instantaneous activities, like maximum is playing major roles in finger selection to operate button.
\end{abstract}

Keywords: neural activities, NIRS, quick components, finger selection, button operation

\section{Introduction}

Working brain needs more oxygen supply. Thus hemodynamic studies expect the areas supplied with rich oxygen are tentatively working. But this is only an expectation, but no evidence. We have to find evidences related to parts of the brain actually functioning. Hemodynamic studies are interested in slow components of NIRS, especially oxyHb. People engaged in hemodynamics, however have realized the existence of quick signal while recording NIRS. So far the signal is regarded as noise, and skill of NIRS measurement is to find the location where the noise can be minimized.

So called noise is so large to be regarded as measurement noise, it must be substantial physiological signal, which we named quick component of NIRS. Attempts have been made to analyze the signal and to make the model of the signal source [5]. Evidence are convincing that the quick component is result of neural activities under recording and successful results are promising in relation to daily human activities, like hand up/down, finger sign, and text entry, etc., to which hemodynamic studies are powerless.

Hemodynamic and neural functions are both significant aspects of brain studies. Cerebral infarction might cause hemodynamic problems, which might result in neural dysfunctions. Rehabilitation is the process of recovering hemodynamics and neural function. Neuro-NIRS is a new significant aspect of NIRS studies. 
Analysis of button operation by NIRS.

\section{Quick components}

\subsection{NIRS coordinates}

Fig. 1 is showing Oxy, Deoxy, and Total Hb ( hemoglobin) recording of 6 channels from NIRS. The upper trace corresponds to oxy, the middle to total, and the lower to deoxy Hb. The channel number is indicated at the middle of the left side of each chart. Looking to the channel 26, oxy and deoxy $\mathrm{Hb}$ contains big high frequency components, which we call quick components, the total $\mathrm{Hb}$ contains less. The traces of 29 and 32 contain less quick components, in both oxy and deoxy, as well as total Hb.

Dynamics of Oxy and deoxy $\mathrm{Hb}$ can be represented by total and difference $\mathrm{Hb}$ as below:

Total $\mathrm{Hb}$ : Total $=0 x y+$ Deoxy

Difference $\mathrm{Hb}$ :

$$
\text { O_D= Oxy -Deoxy }
$$

Fig. 2 is showing traces of above variables. The quick components of $\mathrm{Oxy} \mathrm{Hb}$ and Deoxy $\mathrm{Hb}$ are in opposite phases, thus by the addition of quick components, they are almost compensated each other, while by subtraction, the quick component is almost doubled in amplitude.

Applying moving average of 2 second, quick and slow components of Total and O_D can be derived.

$\begin{array}{ll}\text { Slow total } \mathrm{Hb}: & \text { slow total=Average (Total) } \\ \text { Slow difference } \mathrm{Hb}: & \text { slow O_D=Average (O_D) } \\ \text { Quick total } \mathrm{Hb}: & \text { Quick T= Total }- \text { Average(Total ) } \\ \text { Quick difference } \mathrm{Hb}: & \text { Quick O_D }=\text { O_D }- \text { Average(O_D) }\end{array}$

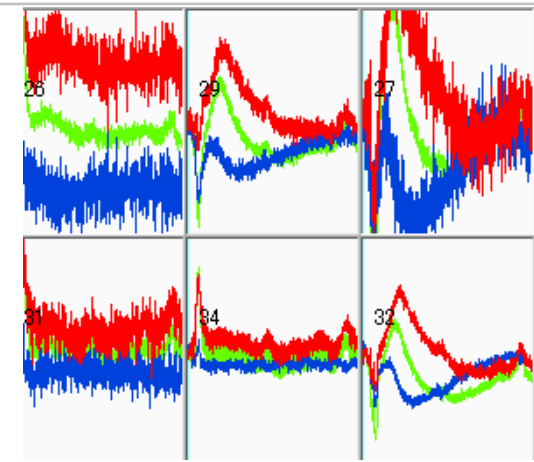

Fig. 1 Raw traces of NIRS

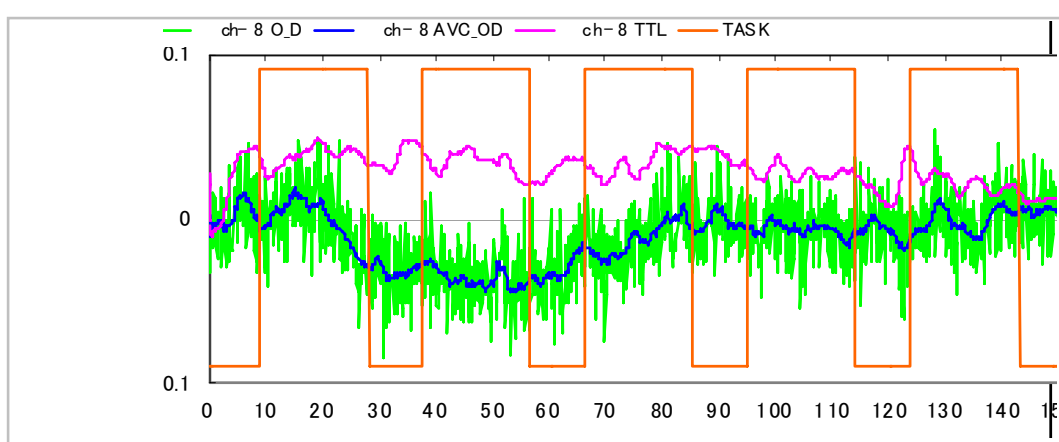

Fig.2 Total $\mathrm{Hb}$ (red) and difference $\mathrm{Hb}$ (green) and the slow O_D (blue) among the middle of difference $\mathrm{Hb}\left(\mathrm{O} \_\mathrm{D}\right)$ trace. Square trace indicates on and off of task. 


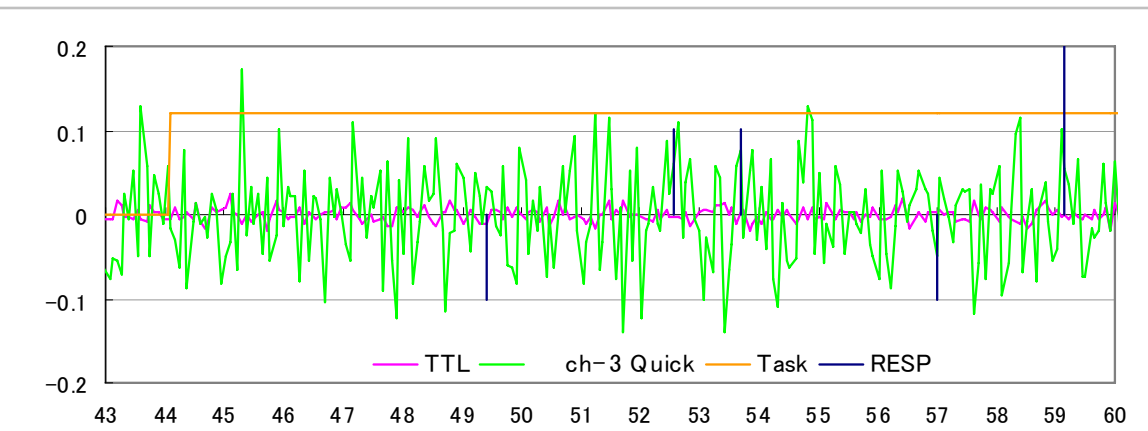

Fig. 3 Traces of Quick O_D and Quick total, sampled at every 0.1 sec.

Thus the analysis of quick components of NIRS can be concentrated to the quick O_D. The quick components derived from eq. (5) and (6) are shown in Fig. 3. The magnitude of quick total is about $1 / 10$ of quick O_D.

\section{Button Selection Task}

\subsection{Experimental Systems}

NIRS (Near Infra-Red Spectroscopy) facility was a product of Shimadzu co. (OMM3000/12) which is installed in Future ITC Research Center, KARC (Kansai Advanced ICT Research Center, Akashi, Kobe, Japan). The authors were honored by Joint Research Treaty with the center to use the NIRS facility. The NIRS had 12-16 light transmitter and receiver pairs and was capable of processing up to 54 channels of brain activities depending on transmitter and receiver arraignment. The arraignment we adopted in measurement is shown in Fig. 4. By this arraignment, 48 channel brain activities are collected at every 0.1 second interval. Beside NIRS data, the machine is capable of 8 external analogue signals, which are used to record start, stop, button operations and task completion signals together with NIRS data.

Experimental task design and the analysis methods are developed by authors. At this moment, the subjects participated in the measurement are graduate students,

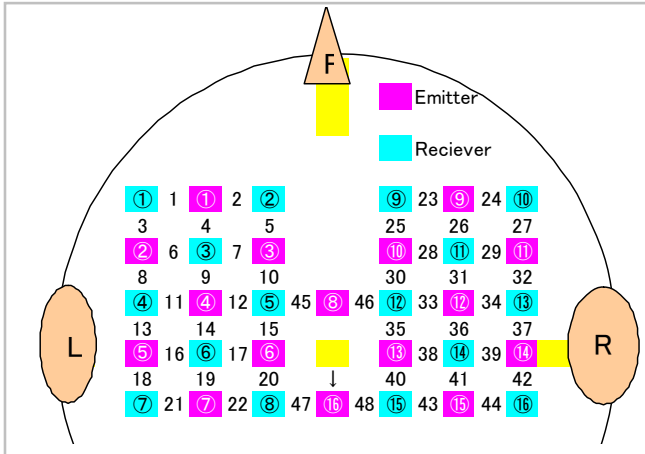

Fig. 4. Transmitter/receiver arraignment 48 channel data are collected at $0.1 \mathrm{sec}$. interval. 
Analysis of button operation by NIRS.

volunteered with informed consent in accordance with the Institutional Review Board of Kobe Women's University. Analysis for larger population should be reported elsewhere.

\subsection{Finger Selection Task (Button Selection)}

Subject rest her hand (left or right) on the button box. Subject operated a button in accordance to the instructions generated by computer and presented either on visual display or by computer reproduced voice. Instructions were to choose a button either one of right, left, up or down. The subject fingers are allocated at home position, in which middle finger stay in the location of center. Fig. 5 shows the case, the box is operated by left hand from left side. In case of up or

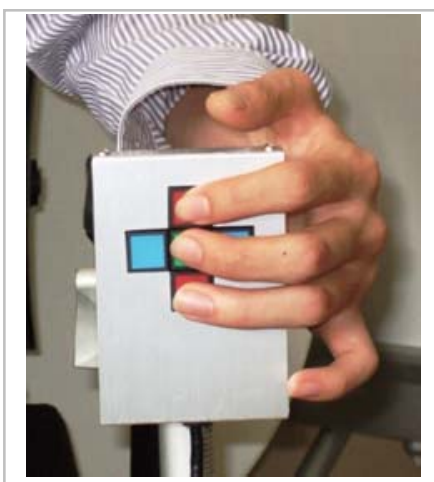

Fig. 5 Button selection by left hand ( side entry) down instruction, index or ring finger might be selected to operate a red button. In case of right or left, middle finger has to change its location by changing the bending angle. The change in necessary action might be related to different brain activities to be analyzed in this paper.

Two methods of visual presentation are used in case of visual display, i.e. by letter (上, 下， 左, 右) and by pictogram ( $\uparrow, \downarrow, \leftarrow, \rightarrow$ ). An instruction to operate is presented once by 3 second in average; the order of instruction is randomized. The instruction is presented on display for 1.1 second. The operator completed the button push tasks almost within 1.1 second. The average response time was in between $0.4-0.8$ second depending on the instruction. The responses were all correct. Analysis of this paper will be limited within the left hand, side entry data (20 trials).

The essential bases of quick component analysis will be explained by applying the method to the button selection data. Total analysis and

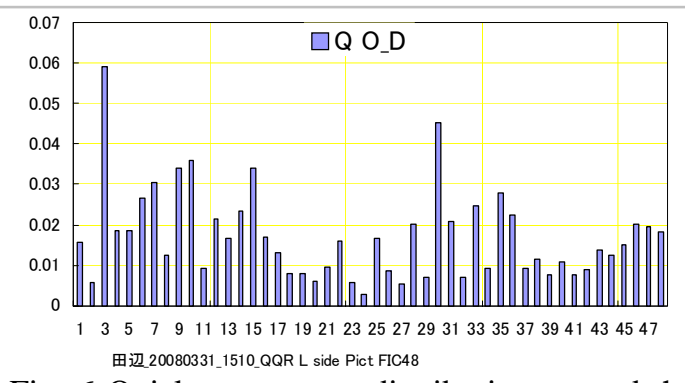

Fig. 6 Quick component distribution around the channels. Ordinate scale in $\mathrm{mMol} / l \cdot \mathrm{cm}$.

comparison of all the data are beyond the scope of this paper. 


\section{Fuchigami: Finger selection}

\subsection{Analysis of Quick Components}

Unified quantitative formulation of quick component is necessary, for the comparison of quick components, derived from various channels at various tasks. An appropriate function applicable to noise like signal as quick components is Avedev ( Microsoft Excel function), which is average of absolute difference of data and their mean value. In our analysis, slow component is subtracted from O_D signal, thus the mean value of quick component is assumed to be zero. In short, avedev is time average of absolute value of quick components.

In the previous papers, long term (>30second) distributions of quick components around different channels and their changes related to tasks are reported [4]. A distribution proper to the case of this paper is shown in Fig. 6, where pictogram is used for instruction display, and operator used left hand at side entry position.

Avedev is stable in value, when averaging interval is taken 10 seconds or longer. It change considerably as averaging interval is shorten, reflecting change of brain activities under the recording. In this paper, a method to evaluate short term change in brain activities is analyzed by introducing two parameters, $\mathrm{M}$ (magnitude) and D (density), related to Q.( avedev of quick component). The relation of these parameters is formulated as below.

$$
Q=M * D
$$

Here

$$
M=\max (\max (\text { member }),-\min (\text { member }))
$$

member: data within averaging interval. Denoting

$\mathrm{N}$ : the number of data in the interval, inequality below is derived.

$$
0<1 / N<D<1
$$

Inequality (9) states that the density remains within the bound of 0 and 1 . Further studies are concentrated to the change of $M$ and $D$, in succession of averaging cycles.

\section{Response Analyses}

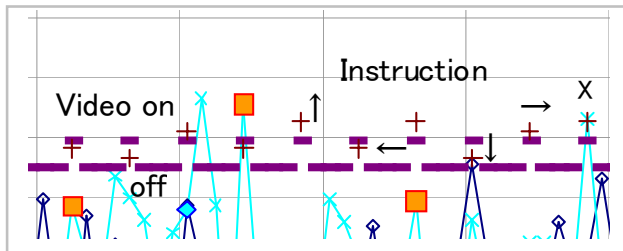

Fig.7a Video on/off (- up/down), button instruction $(\uparrow, \downarrow, \leftarrow, \rightarrow)$ and the button responses $(+)$.
Quick component is analyzed below taking the averaging interval 1.1 second. Some time traces of specific channels are shown in Fig. 7 b) - e). Notations for video on/off and button selection are explained in Fig. 7 a). Instruction display is on when - symbol is in higher position, and while instruction is off, the - symbol is in lower position.

Result of button selection is shown by distance from display interval line (-). 
Analysis of button operation by NIRS.

Instruction is shown by pictogram ( $\uparrow, \downarrow, \leftarrow, \rightarrow$ ) adjacent to corresponding + symbols in Fig. 7a). Symbol $(+)$ touching just above video on sign is correct response to right $(\rightarrow)$ instruction, while one touching just below is corresponding to $(\leftarrow)$ instruction. Symbol $(+)$ above and below apart from video on sign is corresponding to up $(\uparrow)$ and down $(\downarrow)$ instruction.

\subsection{Visual Analysis}

Fig 7b) illustrates -min. and 0.1D (density) of each averaging interval in succession, together with display and response detail while performing button selection task. Blue trace illustrates the -min. Readers might realize many peaks of blue trace are synchronized to the video and the response sign. The peaks synchronized to response signs are illustrated by filled symbol of enlarged size. Time location of synchronization is denoted by letter $\mathrm{N}$.

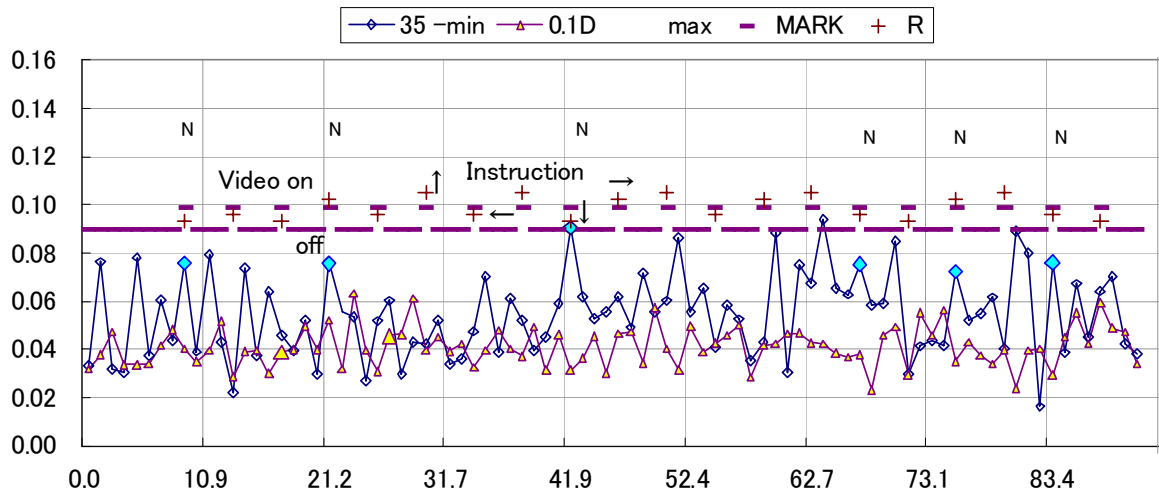

Fig. 7b) Response synchronization of -min. wave form to various instructions, denoted by "N".

In general synchronizations of peaks and responses occur frequently in response to various instructions, but not consistently to a definite instruction. But ch.35 is a specific channel, which shows synchronization to one specific instruction.

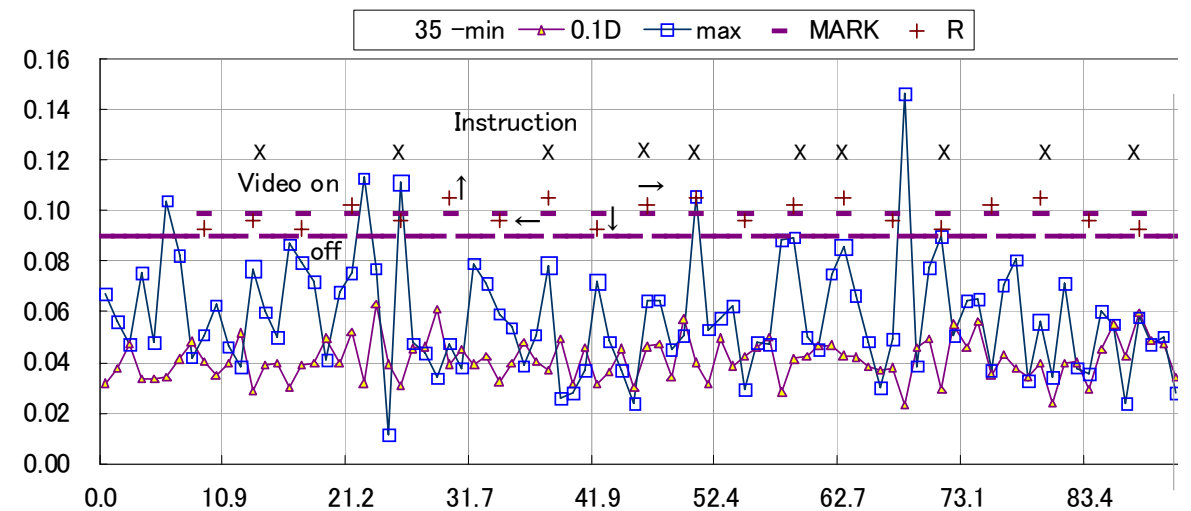

Fig. 7c). Response synchronization of max. wave form to various instructions, denoted by "X". 


\section{Fuchigami: Finger selection}

Fig. 7c). is the similar trace as Fig.7b). The only difference is replacement of - min. by max. waveform. Synchronization also happens with max. Synchronization points are indicated by $X$ marks, and square notations of enlarged size. Total $10 \mathrm{X}$ marks are indicated in the figure. Five of them are in responses to up instructions. All the up instructions are responded by synchronization of max. waveform. This is what we mean by consistency. Certain channel of the brain seems to respond consistently to certain instruction response combination.

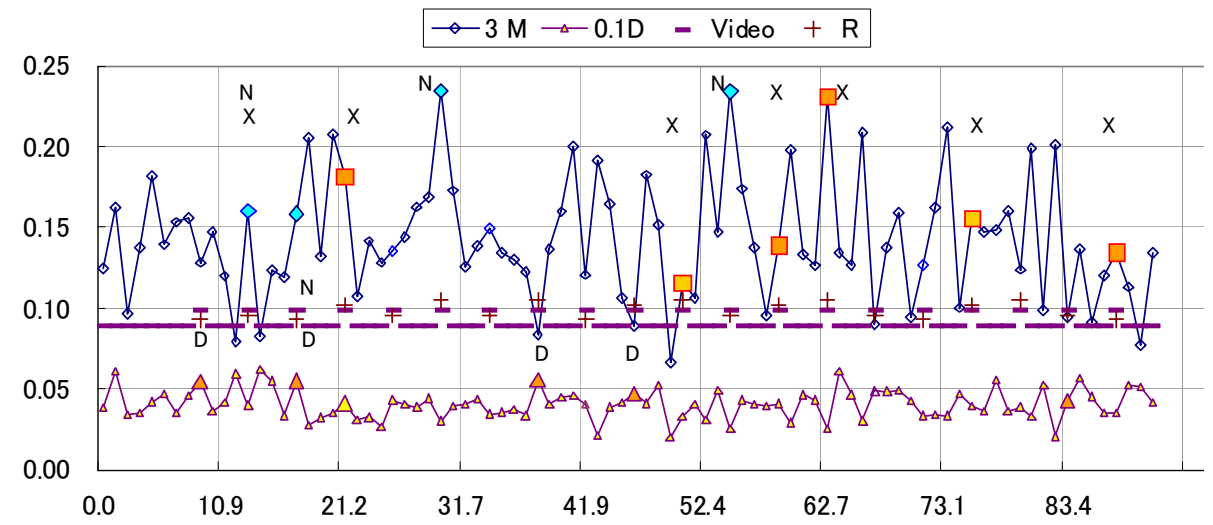

Fig.7d) Response synchronization of magnitude (max. - min) and density (D) wave forms of ch. 3 to various instructions, denoted by " $N, X$ and $D$ ". Double synchronizations $(N+X)$ and $(N+D)$ are identified in time zone between 10.9 and 21.2.

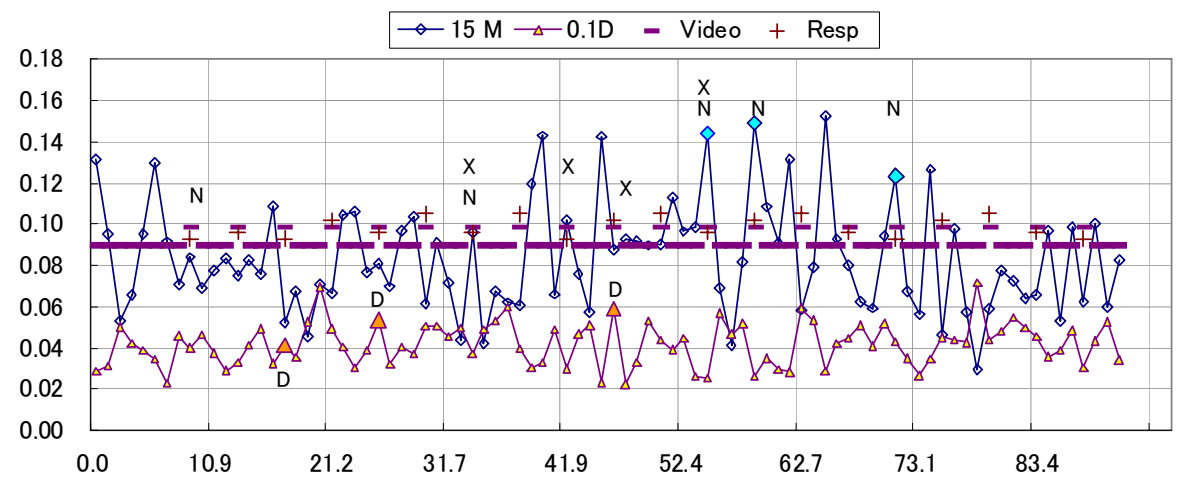

Fig. 7e) Response synchronization of magnitude (max. - min) and density (D) wave forms of ch. 15 to various instructions, denoted by " $N, X$ and D". Double synchronizations, two $(N+X)$ and one $(\mathrm{N}+\mathrm{D})$, are identified in time zone between 31.7 and 62.7 .

Without saying, channels with large magnitude of quick components might show synchronization. It is true. Two major channels, ch. 3, and 15 are selected out from channels of large magnitude of quick components and the synchronization points are shown by colored notation on magnitude trace in Fig.7d) and e). Synchronization happens not always with one waveform. When max. and -min waveforms are close to each other temporarily, double synchronization happens. Double synchronization cannot be indicated by a mark in the graph. Thus synchronization are denoted by text "N" and "X", beside notation mark. In many cases, marks often overlaps with other 
Analysis of button operation by NIRS.

marks, double indications are useful. Double synchronizations also happen between density waveform and max. or -min. Some cases of double synchronization between $\mathrm{N}$ and $\mathrm{X}$, and between $\mathrm{X}$ and $\mathrm{D}$ are shown in Fig. 7d) and in Fig.7e).

Prominent synchronizations are remarkable but consistency is not found in Fig. 7d) and e).

\subsection{Computer Analysis}

Table 1 Channels with large quick components.

\begin{tabular}{|c|c|c|c|c|c|c|c|c|c|}
\hline \multirow{2}{*}{\multicolumn{3}{|c|}{$\mathrm{X} / \mathrm{N}$ Instr TIME }} & \multicolumn{7}{|c|}{ Channel } \\
\hline & & & 3 & 7 & 9 & 10 & 15 & 30 & 35 \\
\hline $\mathrm{N}$ & $\uparrow$ & 29.5 & 23 & & & 5 & & & \\
\hline $\mathrm{N}$ & $\uparrow$ & 37.8 & & & & 14 & & 11 & \\
\hline $\mathrm{N}$ & $\uparrow$ & 50.2 & & 7 & & & & & \\
\hline $\mathrm{N}$ & $\uparrow$ & 62.7 & & & 9 & & & 9 & \\
\hline $\mathrm{N}$ & $\uparrow$ & 79.3 & & 10 & & & & & \\
\hline$X$ & $\uparrow$ & 29.5 & & & & & & & 5 \\
\hline $\mathrm{X}$ & $\uparrow$ & 37.8 & & & 7 & & & & 8 \\
\hline$X$ & $\uparrow$ & 50.2 & 12 & & & & & & 11 \\
\hline$X$ & $\uparrow$ & 62.7 & 23 & & & 8 & & & 9 \\
\hline $\mathrm{X}$ & $\uparrow$ & 79.3 & & & & & & & 6 \\
\hline $\mathrm{N}$ & $\rightarrow$ & 21.2 & & & & & & & 8 \\
\hline $\mathrm{N}$ & $\rightarrow$ & 46.1 & & & & 12 & & & \\
\hline $\mathrm{N}$ & $\rightarrow$ & 58.5 & & 5 & & & 15 & & \\
\hline $\mathrm{N}$ & $\rightarrow$ & 75.1 & & 6 & & & & & 7 \\
\hline$X$ & $\rightarrow$ & 21.2 & 18 & & & & & 17 & \\
\hline $\mathrm{X}$ & $\rightarrow$ & 46.1 & & & 11 & & 9 & & \\
\hline$X$ & $\rightarrow$ & 58.5 & 14 & 9 & & & & & \\
\hline$X$ & $\rightarrow$ & 75.1 & 12 & & & 11 & & & \\
\hline $\mathrm{N}$ & $\downarrow$ & 8.7 & & & & 9 & 8 & & 8 \\
\hline $\mathrm{N}$ & $\downarrow$ & 17 & 14 & & & & & 11 & \\
\hline $\mathrm{N}$ & $\downarrow$ & 41.9 & & & & & & & 9 \\
\hline $\mathrm{N}$ & $\downarrow$ & 70.9 & & 8 & & & 12 & & \\
\hline $\mathrm{N}$ & $\downarrow$ & 87.6 & & 12 & & 16 & & & \\
\hline$X$ & $\downarrow$ & 8.7 & & & 10 & & & & \\
\hline $\mathrm{X}$ & $\downarrow$ & 17 & & 7 & 6 & & & & \\
\hline $\mathrm{X}$ & $\downarrow$ & 41.9 & & & & & 10 & & 7 \\
\hline$X$ & $\downarrow$ & 70.9 & & & 6 & 10 & & & 9 \\
\hline$X$ & $\downarrow$ & 87.6 & 13 & 8 & 10 & 11 & & & 6 \\
\hline $\mathrm{N}$ & $\leftarrow$ & 12.9 & 10 & & & & & 10 & \\
\hline $\mathrm{N}$ & $\leftarrow$ & 25.3 & & & & 12 & & & \\
\hline $\mathrm{N}$ & $\leftarrow$ & 33.6 & & & & & 8 & 12 & \\
\hline $\mathrm{N}$ & $\leftarrow$ & 54.4 & 23 & 6 & & & 10 & 13 & \\
\hline $\mathrm{N}$ & $\leftarrow$ & 66.8 & & 7 & & & & & 8 \\
\hline $\mathrm{N}$ & $\leftarrow$ & 83.4 & & 10 & & & & & 8 \\
\hline$X$ & $\leftarrow$ & 12.9 & 16 & 7 & & & & 8 & 8 \\
\hline$X$ & $\leftarrow$ & 25.3 & & & 8 & 8 & & & 11 \\
\hline$X$ & $\leftarrow$ & 33.6 & & & & & 10 & & \\
\hline$X$ & $\leftarrow$ & 54.4 & & & & & 14 & 12 & \\
\hline$x$ & $\leftarrow$ & 66.8 & & & & & & 11 & \\
\hline$X$ & $\leftarrow$ & 83.4 & & 7 & & 9 & & & \\
\hline
\end{tabular}

For the further minute studies, visual analysis of every one peak is powerless. The computer algorithm, shown below, is applied to1920 data points, which are 48 channels*20 trials*2 wave forms (max,-min).

The results are shown in Table 1 and 2. Table 1 is concerned with 7 channels with big magnitude, and Table 2 is concerned with 18 channels with some consistency. Other 23channels not belonging to either groups are to be mentioned elsewhere.

The consistency is graded in three levels, full $(>80 \%)$, fair $(80 \sim 60 \%)$ and low (<60\%). In Table 1, channel 35 showed full consistency to up ( $\uparrow$ ) instruction. Channel 2 to right $(\rightarrow)$ and 9 down( $\downarrow$ ) showed fair consistency. There were no candidate channels for consistency in $\mathrm{N}$ synchronization.

\begin{tabular}{|c|}
\hline \multicolumn{1}{|c|}{ Algorithm for } \\
synchronization detection \\
$\mathrm{m}=\mathrm{max}$ or -min of current interval \\
$\mathrm{m}_{\mathrm{E}}: \mathrm{m}$ of previous interval \\
$\mathrm{m}_{\mathrm{N}}$ : $\mathrm{m}$ of succeeding interval \\
$\mathrm{m}=\mathrm{IF}\left(\right.$ and $\left(\mathrm{m}^{*} \mathrm{~g}>\mathrm{m}_{\mathrm{E}}, \mathrm{m}^{*} \mathrm{~g}>\mathrm{m}_{\mathrm{N}}\right)$ \\
$\mathrm{m}^{*} 100$, ,") \\
$\mathrm{g}=0.9$ : peak margin \\
In order to avoid decimal point, $\mathrm{m}^{\prime} \mathrm{s}$ \\
in the table are shown after \\
multiplied by 100 .
\end{tabular}


M. Fuchigami: Finger selection

Table 2 Channel group related to response consistency

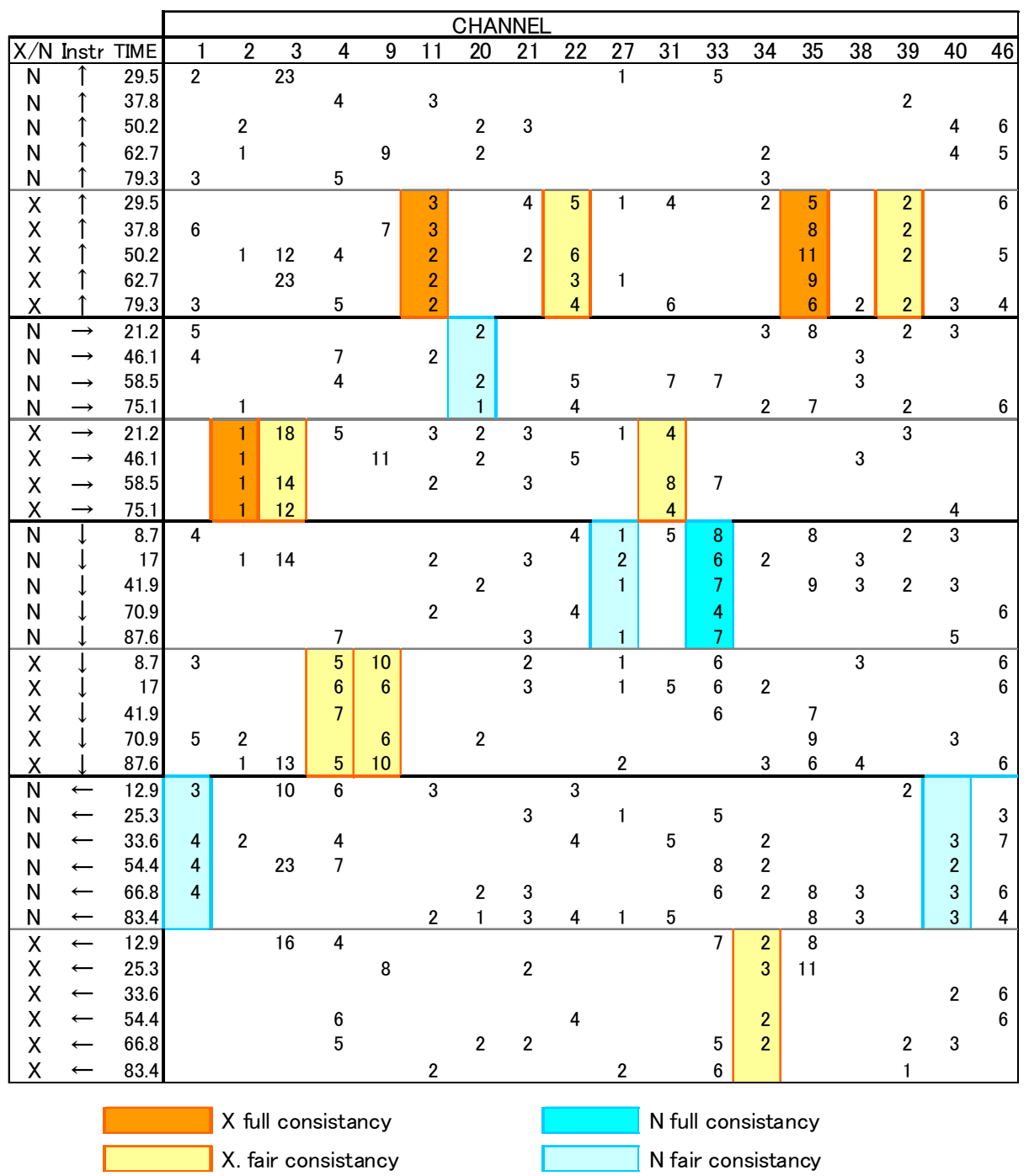

\section{Consistency Channels}

In the Table 2 are collected synchronization distributions of consistent group. First five line from the above are $\mathrm{N}$ synchronization for up instruction( $\uparrow$ ). Full consistency channels are not found in these lines. In the second 5 lines from the above, $\mathrm{X}$ synchronization for up instruction( $\uparrow$ ) shows full consistency in 11 and 35 channels. Fair consistency is also found in 22 and 39 channels. 


\section{Analysis of button operation by NIRS.}

In the third 4 lines from the above, $\mathrm{N}$ synchronization for right $(\rightarrow)$ instructions are shown. Full consistency channels are not found, but fair consistency is found in channel 20. In the next 4 lines from the above, $\mathrm{X}$ synchronization for right $(\rightarrow)$ instructions are shown. Full consistency is found in channel 2, and fair consistency is found in channel 3 and 31 .

In the 5 five lines by the middle of the Table2, $\mathrm{N}$ synchronization for down( $\downarrow$ ) instructions are shown. Full consistency is found in channel 33 and fair consistency is found in channel 27. Next 5 lines below are X synchronization for down $(\downarrow)$ instructions are shown. No full synchronization but two fair synchronization channels are found in channel 4 and 9 . In the bottom of Table 2, $\mathrm{N}$ and $\mathrm{X}$ synchronization for left $(\leftarrow)$ instruction are shown. No full consistency channels but fair consistency is found in some channels.

One question is why no full consistency for left $(\leftarrow)$ instruction. It may be related to the experimental set. This experiment used the left hand, side entry task as explained in chapter 3. In this set up, to touch left and right button middle finger of left hand has to be extended or constricted. These actions will be more complex compared to up and down action which is simple touching to a button. The effects of different finger selection and actions can be examined by changing hand rest position, and by comparing results of left and right hands.

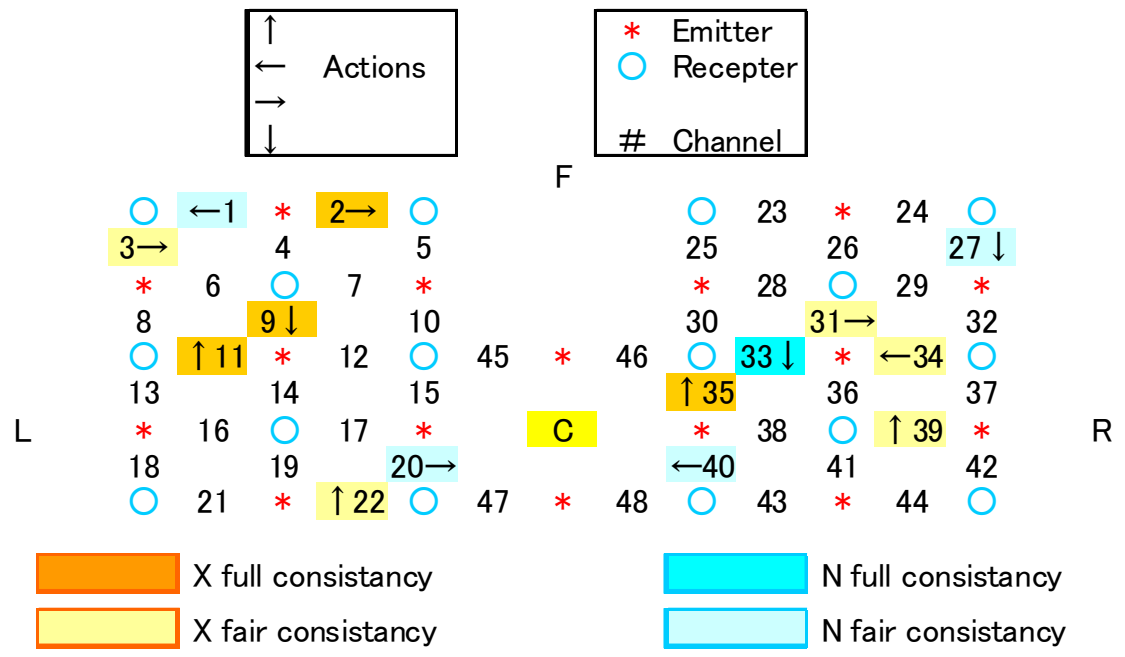

Fig.8 Allocation of full and fair consistency channels for left hand side entry operation. Instruction and action direction are indicated by arrows.

In conclusion of synchronization analysis, consistency allocation for the left hand side entry operation is illustrated in Fig. 8.

Table 1 is channel group with active quick components and effecting to almost all activities but not always specifically related to certain tasks. Table 2 is the channel group which are not always active in quick component but certain consistency to finger selection is confirm by this analysis. Some more channels are supposed to be included to Table 2 group based on further analysis related to left/right hand and top/side entry variations. Still more channels are expected 


\section{Fuchigami: Finger selection}

to enter the group based on instruction presentation variations. At the moment 23 more channels remain out of either group.

Further analysis is done with the density related to finger selection. The NIRS analysis[7] of continuous events so far suggests that the channels actually working are less variable, so that standard deviations are small, while channels out of work show large deviation. Similar trend are observed also in finger selections task.

\section{Conclusion}

Button operation by finger is still most essential activities supporting human computer interactions, to which not much is known about the brain functions.

Quick component analysis is successfully applied to finger selection of button operation task.

Magnitude and density analysis will extend the scope of brain function studies to real time activities.

\section{References}

[1] Tamura, H., Omori, M., Choui, M.: NIRS trajectories in Oxy-Deoxy Hb plane and the trajectory map to understand brain activities related to human interface, pp. 994-1003, Proc. HCII 2007, Springer-Verlag, 2007/7

[2] Fuchigami, M., Okada, A., Tamura, H., Omori, M.: A Comparative Study of Brain Activities Engaged in Interface Operations by Means of NIRS Trajectory Map, pp. 830-839, Proc. HCII 2007/7, Springer-Verlag 2007.

[3] Tamura, H., Fuchigami M., Okada A., Omori M., Hasegawa S., Fujikake K., Choui M. : On Physiological Roles of Quick Components in Near Infrared Spectroscopy (in Japanese), Proc. Mobile Interactions’08, pp. 97- 102, Society of Mobile Interactions, 2008/7

[4] Tamura, H., Fuchigami M., Okada A.,: On Physiological Roles of Quick Components in Near Infrared Spectroscopy (in Japanese), Proc. Mobile Interactions’08, pp. 97- 102, Society of Mobile Interactions, 2008/7

[5] Tamura, H., Fuchigami M., Okada, A.: Analysis of Text Entry behavior by NIRS Quick Components, Proc. Mobile Interactions'09, pp. 109-114, Society of Mobile Interactions, 2009/3

[6] Fuchigami M., Okada, A., Tamura, H.,: Analysis of NIRS Quick Components in directionselective button operations, Proc. Mobile Interactions’09, pp. 115-120, Society of Mobile Interactions, 2009/3

[7] Tamura, H., Fuchigami, M., Okada, A., Neuro-NIRS: Analysis of Neural Activities using NIRS, HCII2009 